\title{
T-cell line for HIV drug screening using EGFP as a quantitative marker of HIV-1 replication
}

\author{
Christina Ochsenbauer-Jambor, Jennifer Jones, Marintha Heil, Kenneth P. Zammit, and Olaf Kutsch
}

BioTechniques 40:91-100 (January 2006)

doi $10.2144 / 000112072$

\begin{abstract}
The rapid increase of viral strains that are resistant to the currently available antiretroviral drugs is a threat to the success of current human immunodeficiency virus type 1 (HIV-1) treatment and emphasizes the importance of developing novel anti-HIV-1 compounds. To improve the current abilities to screen for novel HIV-1 inhibitors, here we introduce a T-cell-based reporter cell line (JLTRG-R5) that expresses both HIV-1 coreceptors, CXCR4 and CCR5, and provides the convenience of using enhanced green fluorescent protein (EGFP) as a direct and quantitative marker. Unlike previous EGFP-based reporter cell lines, JLTRG-R5 cells have an unusually high dynamic signal range, sufficient for plate reader detection using a 384-well format. In this format, JLTRG$R 5$ cell-based infectivity assays have a $Z^{\prime}$-factor of 0.78 , which defines the assay as extremely robust and clearly amenable to highthroughput screening. The functional similarity of the JLTRG-R5 cell line and peripheral blood mononuclear cells (PBMCs) was demonstrated through the identity of the inhibitory concentrations, $50 \%\left(I_{50} s\right)$ for four antiretroviral compounds or neutralizing antibodies. Because EGFP can be directly and continuously quantified in cell culture, the reporter cell line requires no manipulation during assay preparation or analysis. In addition, the EGFP marker allows for data acquisition at an optimal time point by prescreening selected positive control wells using fluorescent microscopy. These characteristics make the system extremely flexible, rapid, and inexpensive. Due to its intrinsic flexibility, the JLTRG-R5 cell-based reporter system provides a powerful tool to greatly facilitate future screening for HIV-1 inhibitors.
\end{abstract}

\section{INTRODUCTION}

Despite its tremendous benefits, highly active antiretroviral therapy (HAART) is not able to eradicate human immunodeficiency virus (HIV) from treated patients (1). In this scenario, the continuously increasing number of viral strains that are resistant to therapy, as well as the adverse drug side effects that are frequently associated with long-term treatment, pose a serious threat to the current treatment success and require the development of new anti-HIV type 1 (HIV-1) compounds (2). In particular, the recent therapeutic success of T-20, a peptide inhibiting viral entry, has highlighted the general potential of HIV-1 entry/fusion inhibitors to complement current highly active antiretroviral therapy. While T20 is an example of successful rational drug design, other compounds such as ALX 40-4C, a nonpeptide molecule inhibiting HIV-1 entry by targeting the HIV-1 coreceptor CXCR4, demonstrate the limitations of this approach: ALX 40-4C was initially designed to inhibit HIV-1 Tat activity, but in vitro experiments revealed that its mode of action was the inhibition of viral entry $(3,4)$. Despite the tremendous advances rational drug design has brought to the field, such examples demonstrate the particular value of cell-based highthroughput screening (HTS) assays. For this purpose, a variety of cell lines and reporter assays that are suitable for HTS to various degrees have been established (5-9). Suitability is mostly defined by the costs of the actual screening and the time required for the assay. To justify the screening of an industrial-size compound library, which can hold several million compounds, the screening costs per compound need to be minimized. An ideal reporter cell line to screen for inhibitors of HIV-1 replication thus needs to fulfill several requirements: $(i)$ the reporter cell line should be an immortalized T-cell line expressing both HIV-1 coreceptors, CXCR4 and CCR5, and (ii) the reporter cell line should provide a stable and quantifiable marker for HIV1 expression, such as secreted alkaline phosphatase (SEAP), luciferase, or enhanced green fluorescent protein (EGFP) to avoid the costly and timeconsuming direct detection and quantification of viral gene products [e.g., HIV-1 p24 enzyme-linked immunosorbent assay (ELISA) and reverse transcription assay]. Two reporter cell lines, MOCHA (10) and CEM. NKR-CCR5-Luc (11), which meet these criteria were recently published. The cell lines use SEAP or luciferase, respectively, under the control of an HIV-1 long terminal repeat (LTR) as the indicator of HIV-1 expression. However, during assay analysis, these cell lines still require time-consuming or relatively costly manipulation of the cell culture (cell lysis, supernatant 
Table 1. IC ${ }_{50}$ Values of Tested HIV-1 Inhibitors

\begin{tabular}{|cccc|}
\hline Inhibitor & JLTRG-R5 & PBMCs & Published (Reference) \\
\hline 3TC & $0.03 \mu \mathrm{M}^{\mathrm{a}}$ & $0.06 \mu \mathrm{M}^{\mathrm{a}}$ & $0.07 \mu \mathrm{M}(16)$ \\
Indinavir & $0.003 \mu \mathrm{M}^{\mathrm{a}}$ & $0.01 \mu \mathrm{M}^{\mathrm{a}}$ & $\mathrm{IC}_{95} 0.1 \mu \mathrm{M}(17)$ \\
T-20 & $0.006 \mu \mathrm{g} / \mathrm{mL}^{\mathrm{b}}$ & $1.2 \mu \mathrm{g} / \mathrm{mL}^{\mathrm{m}}$ & $0.002-0.015 \mu \mathrm{g} / \mathrm{mL}^{\mathrm{d}}$ \\
& $0.005 \mu \mathrm{g} / \mathrm{mL}^{\mathrm{c}}$ & & $1.2 \mu \mathrm{g} / \mathrm{mL}^{\mathrm{e}}(21)$ \\
TAK-779 & $0.005 \mu \mathrm{M}^{\mathrm{c}}$ & $0.005 \mu \mathrm{M}^{\mathrm{c}}$ & $0.004 \mu \mathrm{M}^{\mathrm{c}}(21)$ \\
IC & & \\
aHIV-1 NL4-3 & & \\
bHIV-1 NL4-3(GIV) & & & \\
cHIV-1 BAL \\
dCEM cells \\
ePBMCs
\end{tabular}

transfer, and the addition of chemicals) to obtain the final readout.

Here we demonstrate that, based on a stably integrated LTR-EGFP reporter construct, HIV-1 expression in the JTRG-R5 reporter cell line can be directly detected and quantified in cell culture without additional manipulation. This not only significantly reduces the costs of any HTS effort but also provides the opportunity for the direct kinetic analysis of drug effects on HIV-1 expression over an extended time period. In addition, the cell line allows for single-cell analysis using flow cytometry. Using EGFP as a direct marker of HIV-1 infection, follow-up experiments that define the characteristics of an identified lead compound can then be performed with great convenience. If compound availability is low, cells from wells with positive drug hits identified during a drug screen can be removed from the plate and directly subjected to flow cytometry to account for potential cytotoxic or apoptotic effects of the tested compound. We demonstrate that JLTRG-R5 cells can be used in a 384well plate-based format and quantitatively reflect previous findings obtained from peripheral blood mononuclear cell (PBMC) cultures. Thus, the cell line improves existing methodologies to identify novel anti-HIV-1 compounds.

\section{MATERIALS AND METHODS}

\section{Cell Culture and Reagents}

The reporter T-cell lines JLTRG and JLTRG-R5 were maintained at an average cell density of $0.5 \times 10^{6}$ cells/mL in RPMI 1640 (Mediatech,
Herndon, VA, USA) supplemented with $2 \mathrm{mM}$ L-glutamine, $100 \mathrm{U} / \mathrm{mL}$ penicillin, $100 \mu \mathrm{g} / \mathrm{mL}$ streptomycin, and $10 \%$ heat-inactivated fetal bovine serum (FBS; HyClone, Logan, UT, USA) (12). The cell lines are identical with respect to CD4 and CXCR4 expression, but only JLTRG-R5 cells express CCR5. We found that CCR5 expression on JLTRG-R5 cells was relatively stable in long-term culture. Over a two-year culture period, only $30 \%$ of the cells lost CCR5 expression. Complete CCR5 expression on a population basis can be easily reestablished by enriching CCR5-positive cells using anti-CCR5 antibody-coated magnetic beads (Dynal Biotech, Lake Success, NY, USA) or by fluorescenceactivated cell sorting techniques. Prior to the infection experiments, the cells were split to $1 \times 10^{5}$ cells $/ \mathrm{mL}$ and then grown to a density of $5 \times 10^{5}$ cells $/ \mathrm{mL}$ to assure optimal susceptibility to HIV1 infection. JC53BL-13 cells (TZMBL) were cultured and infected as previously described (13). Briefly, the cells were maintained in Dulbecco's modified Eagle's medium (DMEM; Mediatech) supplemented with $2 \mathrm{mM}$ L-glutamine, $100 \mathrm{U} / \mathrm{mL}$ penicillin, 100 $\mu \mathrm{g} / \mathrm{mL}$ streptomycin, and $10 \%$ heatinactivated FBS.

PBMCs used to generate infectious viral supernatants were isolated from the blood of healthy donors by FicollPaque $^{\mathrm{TM}}$ density gradient centrifugation (Amersham Biosciences, Uppsala, Sweden) and were cultured in RPMI 1640 supplemented with $10 \%$ heatinactivated FBS, $2 \mathrm{mM}$ L-glutamine, $100 \mathrm{U} / \mathrm{mL}$ penicillin, and $100 \mu \mathrm{g} / \mathrm{mL}$ streptomycin. PBMCs were initially PHA/interleukin-2 stimulated and infected with the various HIV-1 patient isolates and HIV-1 clones 4 days following stimulation. All antibodies were purchased from BD Pharmingen (San Diego, CA, USA). PHA-L was obtained from Sigma (St. Louis, MO, USA), and IL-2 was purchased from Biosource International (Camarillo, CA, USA).

\section{Generation of Infectious Viral Supernatants and Patient Sera}

The following HIV-1 isolates or laboratory clones were used during the experiments: HIV-1 CUCY, HIV-1 WEAU, HIV-1 HVH, HIV-1 R8, HIV1 JOMA, HIV-1 Du151, HIV-1 TV1, HIV-1 BAL, HIV-1 SF162, HIV-1 Bx08, and HIV-1 NL4-3. Of these, the HIV-1 isolates CUCY, WEAU, and HVH were R5/X4 viruses [all clade B; University of Alabama Center For Aids Research (UAB CFAR) Repository, Birmingham, AL, USA]. JOMA (group O), TV1 (clade C), and R8 (clade B) were R5 viruses (UAB CFAR Repository). The isolates Bx08 (clade B) and Du151 (clade C) were a kind gift from David Montefiori (Duke University, Durham, NC, USA) and were R5 viruses. In all cases, informed consent was obtained from the respective patients that allowed usage of the samples for research purposes. HIV-1 NL4-3, HIV-1 SF162, and HIV-1 BAL were obtained from the National Institutes of Health (NIH; Bethesda, MD, USA) AIDS Research and Reference Reagent Program (www.aidsreagent.org). All primary patient isolates were propagated in PBMC cultures using phenol red-free RPMI 1640 supplemented with 5\% heat-inactivated FBS, $2 \mathrm{mM}$ L-glutamine, $100 \mathrm{U} / \mathrm{mL}$ penicillin, 100 $\mu \mathrm{g} / \mathrm{mL}$ streptomycin, and $50 \mathrm{U} / \mathrm{mL}$ IL-2. Supernatants were harvested continuously between days 4 and 7 following infection and adjusted for the experiments by either $\mathrm{p} 24$ concentration or infectious units as determined on JLTRG-R5 cells.

\section{Flow Cytometric Analysis}

Flow cytometric analysis was performed using a FACScan ${ }^{\mathrm{TM}}$ and CellQuest $^{\mathrm{TM}}$ software or an LSR II flow cytometer and FACSDiva ${ }^{\mathrm{TM}}$ software (all from BD Biosciences, San Jose, 
A

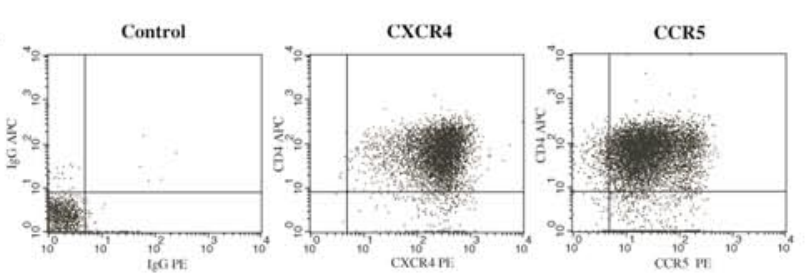

B
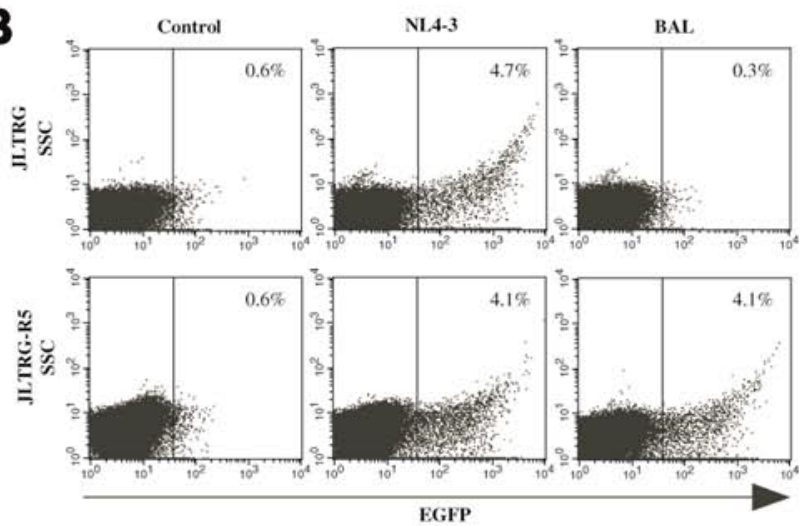

C

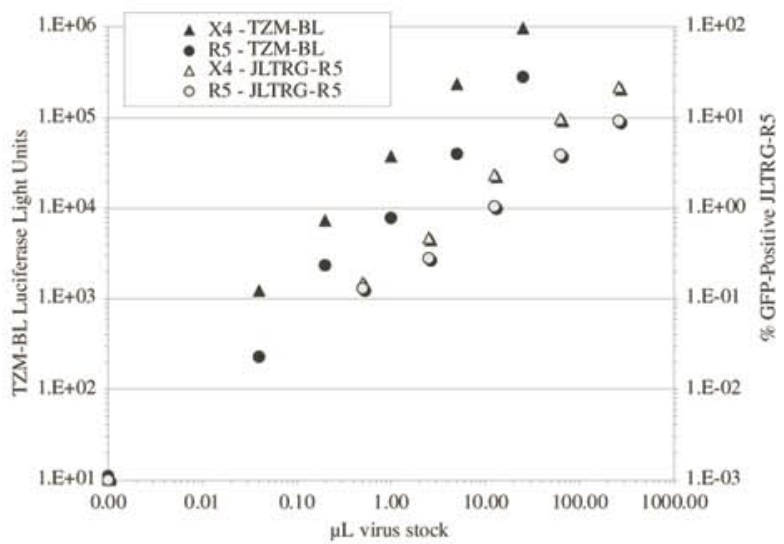

D

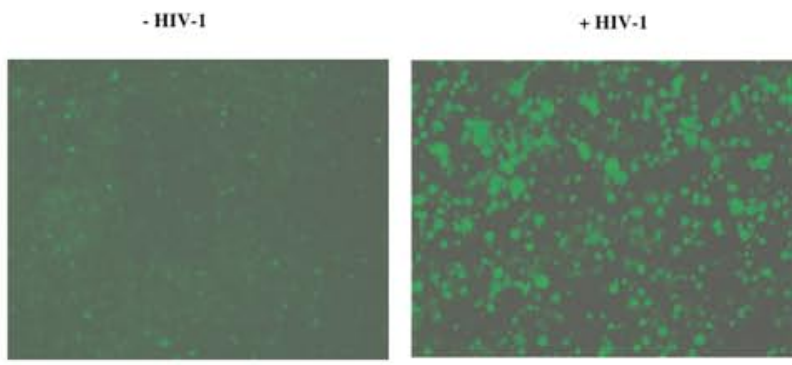

Figure 1. Characterization of JLTRG-R5 cells. (A) JLTRG-R5 cells were stained with isotypematched control antibodies, CD4-APC and CXCR4-PE, and CD4-APC and CCR5-PE to determine the expression levels of the HIV-1 receptor CD4 and the coreceptors CCR5 and CXCR4, respectively. (B) The parental JLTRG cells (CCR5-negative) and JLTRG-R5 cells were infected at an MOI of 0.01 with the X4 virus NL4-3 and the R5 virus BAL. Three days postinfection, the level of HIV-1 replication was determined at the single-cell level using flow cytometric analysis for EGFP. The percentage of EGFPpositive, and thus HIV-1-infected cells, is given for each experimental condition. The results are representative for two independent experiments. (C) Stocks of two isogenic NL4-3-derived viruses encoding patient-derived X4 and R5 env genes, respectively, were titrated on JLTRG-R5 and TZM-BL $\left(4 \times 10^{4}\right.$ cells per 24-well plate). Three days postinfection, the level of HIV-1 replication was determined by flow cytometric analysis for EGFP for JLTRG-R5 cells, and by luciferase activity in cell lysates for TZM-BL cells. (D) Uninfected JLTRG-R5 cells (- HIV-1) or HIV-1-infected JLTRG-R5 cells (+ HIV-1) as visualized by fluorescence microscopy. HIV-1, human immunodeficiency virus type 1; MOI, multiplicity of infection; EGFP, enhanced green fluorescent protein; IgG, immunoglobulin G.
CA, USA). If the cells were not stained for the expression of cell surface markers, all samples were treated with propidium iodide (Sigma) to allow for the exclusion of dead cells during data analysis following standard procedures (14).

\section{4-Well Plate-Based Assays}

All plate-based experiments were performed in 384-well optical bottom black wall plates (Nalge Nunc International, Rochester, NY, USA) and designed to obtain a final cell density of $1 \times 10^{6}$ cells $/ \mathrm{mL}$ in a final volume of $90 \mu \mathrm{L}$ phenol red-free RPMI 1640 per well. This optimal number was obtained by titrating JLTRG-R5 cells over a range of cell numbers per well $\left(1 \times 10^{3}\right.$ to $1 \times 10^{6}$ cell/well $)$ and infecting the cells with HIV-1 NL4-3, followed by plate-based fluorometry 3-6 days postinfection. The phenol red-free RPMI 1640 used in all experiments was supplemented with $2 \mathrm{mM}$ L-glutamine, $100 \mathrm{U} / \mathrm{mL}$ penicillin, $100 \mu \mathrm{g} / \mathrm{mL}$ streptomycin, and $2 \%$ heat-inactivated FBS. Infections were performed in the absence or presence of $4 \mu \mathrm{g} / \mathrm{mL}$ diethylaminoethyl (DEAE)dextran (molecular weight: 5000), which on average resulted in a $20 \%$ increase in the obtained signal. The absence or presence of DEAE-dextran did not alter the quantitative ability of patient sera, TAK-779, or T-20 to neutralize HIV-1 infection. Analysis was performed using a Synergy ${ }^{\mathrm{TM}}$ HT Multi-Detection Microplate Reader (Bio-Tek Instruments, Winooski, VT, USA), equipped with the following filter set: excitation, $488 / 20 \mathrm{~nm}$; emission, $525 / 20 \mathrm{~nm}$.

To determine the $Z^{\prime}$-factor, JLTRG$\mathrm{R} 5$ cells were adjusted to a cell density of $2 \times 10^{6}$ cells $/ \mathrm{mL}$ in phenol red-free RPMI 1640 supplemented with $2 \%$ FBS, of which $50 \mu \mathrm{L}$ were loaded per well. The addition of $50 \mu \mathrm{L}$ of infectious viral cell culture supernatants per well [equal to a multiplicity of infection (MOI) of 0.1$]$ or $50 \mu \mathrm{L}$ of RPMI supplemented with $2 \%$ FBS resulted in a final cell density of 100,000 cells/ well in a total volume of $100 \mu \mathrm{L}$ (or $1 \times$ $10^{6}$ cells $/ \mathrm{mL}$ ). 
Inhibition of Infection by

Antiretroviral Compounds and Neutralizing Antibodies

To determine the inhibitory concentration, $50 \%\left(\mathrm{IC}_{50}\right)$ of the HIV-1 reverse transcriptase inhibitor AZT, JLTRG-R5 cells were adjusted to a cell density of $2.5 \times 10^{6}$ cells $/ \mathrm{mL}$, and $40 \mu \mathrm{L}$ of this cell suspension were loaded into each well. The drug was then added at the various concentrations indicated in a volume of $10 \mu \mathrm{L}$ and the cultures were infected with $50 \mu \mathrm{L}$ of the respective viral supernatants, adjusted to give an MOI of 0.1. The 384-well plates were monitored for EGFP expression every $24 \mathrm{~h}$ for a total of $96 \mathrm{~h}$.

The $\mathrm{IC}_{50}$ of the neutralizing capacity of the patient sera samples (UAB CFAR Repository) was determined by the addition of 0.01 to $10 \mu \mathrm{L}$ of the various patient sera and sera from a noninfected patient, adjusted to a total volume of 10 $\mu \mathrm{L}$ using phenol red-free RPMI, to 50 $\mu \mathrm{L}$ of the viral supernatants. Following $1 \mathrm{~h}$ preincubation, the sera/virus supernatant samples were added to JLTRGR5 cells, which were loaded into the plate in $40 \mu \mathrm{L}$ at a cell density of 2.5 $\times 10^{6}$ cells $/ \mathrm{mL}$. To adjust for a possible influence of the patient serum on the fluorescent signal of the samples, we loaded wells with cells and the various amounts of patient samples but added $50 \mu \mathrm{L}$ RPMI. The cultures were then monitored for EGFP expression every $24 \mathrm{~h}$ for a total of $96 \mathrm{~h}$.

\section{RESULTS}

\section{Establishment of JLTRG-R5 Cells}

Most T-cell lines, including Jurkat cells, exclusively express CXCR4, limiting the permissiveness of these cells to infection with T-cell-tropic or dual-tropic virus clones/isolates, such as HIV-1 NL4-3 or HIV-1 WEAU, respectively (13). Because most viruses isolated from patients use the CCR5 coreceptor, a useful reporter cell line for the discovery of HIV-1 antiretroviral compounds and neutralizing antibodies should express CCR5. To develop a reporter cell line that would allow for HTS for X4, X4/R5, and R5 HIV-1 viruses, we established a naturally CCR5-expressing JLTRG cell clone, a reporter cell line that we have previously established in our laboratory (12). CCR5 expression levels in these cells are similar to CCR5 expression levels seen in PBMCs, but lower than in the fibroblast-based reporter cell line JC53, which is extremely susceptible to HIV-1 infection with R5 viruses (data not shown). Briefly, Jurkat cells had been stably transfected with an LTR-EGFP reporter construct and cloned, and a cell line was selected that expressed minimal levels of basal EGFP in an uninfected state, but responded to HIV-1 infection with extremely high levels of EGFP fluorescence. Clones were screened for high susceptibility to HIV-1 infection with both HIV-1 X4 and HIV-1 R5 strains. The cell line with the most desirable phenotype was named JLTRG-R5 and was chosen for further characterization. The data presented in Figure 1A show that JLTRG-R5 express high levels of CD4, CXCR4, and CCR5. JLTRG-R5 expressed similar levels of CD4 and CXCR4 relative to JLTRG (data not shown).

When infected with HIV-1 NL4-3 or HIV-1 BAL, two prototypic X4 or R5 strains, both cell lines became infected to a similar extent with the CXCR4tropic HIV-1 NL4-3 as determined by flow cytometry. However, only JLTRGR5 cells were susceptible to infection with the CCR5-tropic HIV-1 BAL, a result of the exclusive and distinct expression of CCR5 in JLTRG-R5 cells. The cells were infected at a low MOI of 0.01 to avoid syncytia formation that would bias flow cytometric analysis (Figure 1B).

We compared the relative susceptibility of JLTRG-R5 cells to infection with $\mathrm{X} 4$ and R5 HIV-1 to that of the established HeLa-derived indicator cell line JC53 (TZM-BL) (15), which is highly susceptible to both T-cell-tropic and macrophage-tropic HIV1. As depicted in Figure $1 \mathrm{C}$, two isogenic viruses derived from NL4-3, but encoding patient-derived X4 and R5 env genes, respectively, were titrated on both cell lines. The dose-response of reporter gene expression (luciferase activity for JC53 and EGFP-positive JLTRG-R5 cells in flow cytometry) induced by the respective X4 versus R5 HIV-1 infection was nearly identical in the JLTRG-R5 cell line and was slightly biased against R5 virus in JC53 cells. JLTRG-R5 cells were slightly less sensitive to infection; however, because the cells had been designed for HTS assays and not to determine viral fitness, this poses no actual shortcoming with respect to the intended purpose. Furthermore, Figure 1D demonstrates that fluorescence microscopy is a convenient method to observe the level of HIV-1 infection in JLTRG-R5 cells and can be used to determine the optimal time point to perform assay analysis.

\section{Susceptibility of JLTRG-R5 Cells to Infection with Various HIV-1 Isolates}

In addition to being utilized as a reporter cell line optimized for highthroughput drug screening, JLTRG$R 5$ cells could be used in verification assays if they can be efficiently infected by a selection of divergent HIV-1 strains. To test this possibility, JLTRG-R5 cells were infected with a

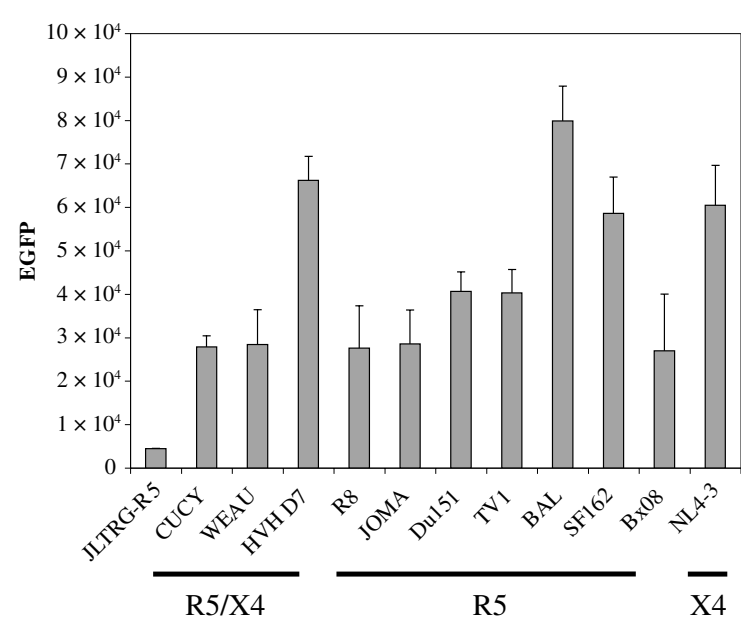

Figure 2. Susceptibility of JLTRG-R5 to infection with various X4, X4/R5, and R5 HIV-1 isolates. JLTRG-R5 cells $\left(1 \times 10^{5}\right)$ were seeded per 384-well plate and infected with the various HIV-1 isolates indicated. After 3 days, the level of HIV-1 infection was determined using plate-based fluorometry. The results represent the mean \pm SD of three independent experiments. HIV-1, human immunodeficiency virus type 1; GFP, green fluorescent protein. 
Equation 1. Mathematical derivation of the $Z^{\prime}$ 'factor and qualitative interpretation.

$$
\begin{aligned}
& Z^{\prime}=1-\frac{\left(3 \sigma_{c+}+3 \sigma_{c}\right)}{\left|\mu_{c+}-\mu_{c-}\right|} \\
& \sigma_{\mathrm{ct}}: \text { standard deviation of } \\
& \text { positive samples } \\
& \sigma_{\text {c }} \text { : standard deviation of } \\
& \text { background samples } \\
& \mu_{\mathrm{c}+} \text { : mean of positive samples } \\
& \begin{array}{l}
\mu_{\mathrm{c}+}: \text { mean of positive samples } \\
\mu_{\mathrm{c}} \text { : } \text { mean of positive samples }
\end{array} \\
& 1=Z^{\prime} \quad \text { ideal assay } \\
& 1>Z^{\prime} \bullet 0.5 \text { excellent assay } \\
& 0.5>Z^{\prime}>0 \text { double assay } \\
& 0=Z^{\prime} \quad \text { "yes/no" type assay } \\
& \mathrm{Z}^{\prime}<0 \quad \text { assay unreliable }
\end{aligned}
$$

panel of viruses with varying receptor affinities, and EGFP fluorescence was determined 3 days postinfection using plate-based fluorometry (Figure 2). We found that all strains tested were capable of efficiently infecting JLTRGR5 cells, and the resulting fluorescent signals ranged between 6 - and 20-fold over control cells as measured using a plate-based 384-well format. The results indicate that JLTRG-R5 cells express sufficient levels of functional HIV-1 receptors to allow for infection with a wide array of HIV-1 primary isolates, making them a useful tool for drug verification.

\section{Characteristics of HIV-1 Replication in JLTRG-R5 Cells in the Presence of Reverse Transcriptase and Protease Inhibitors}

To test whether JLTRG-R5 cells can be used to screen for various types of HIV-1 inhibitors and would reflect findings in PBMCs, we tested the ability of the established HIV-1 reverse transcriptase inhibitor 3TC and of the HIV-1 protease inhibitor indinavir to inhibit HIV-1 replication. For this purpose, we titrated all drugs over a wide concentration range onto JLTRG-R5 cells and then infected the cells with either HIV-1 NL4-3 or HIV-1 BAL. Infection was performed at a relatively low MOI (0.01) to avoid syncytia formation that could not be quantitatively accounted for during flow cytometric analysis. Three days postinfection, the level of infection was determined by flow cytometry using EGFP expression as a readout. The $\mathrm{IC}_{50}$ for 3TC was determined as $0.03 \mu \mathrm{M}$, which reflects previous findings using HIV-1-infected PBMCs, for which the $\mathrm{IC}_{50}$ was determined to be 0.07 $\mu \mathrm{M}$ (16). For indinavir, the $\mathrm{IC}_{50}$ was determined to be $0.003 \mu \mathrm{M}\left(\mathrm{IC}_{95}=0.1\right.$ $\mu \mathrm{M})$, which also closely corresponds to previously reported concentrations for HIV-1 inhibition on PBMCs ( $\mathrm{IC}_{95}$ $=0.1 \mu \mathrm{M}$; Table 1) (17). Single-cell analysis by flow cytometry in this case allowed us to directly determine the level of baseline infection (Figure 3, dashed line) and of the ongoing viral replication during the experimental time frame.

\section{Optimization of JLTRG-R5 Cells for Use in a 384-Well Format}

For HTS to be effective, the system must produce results in an efficient platform such as a 384-well plate. We initially optimized the optimal cell density per well in this format, which we found to be $1 \times 10^{5}$ cells/well. To determine whether JLTRG-R5 cells could be used for HTS, we performed a $Z^{\prime}$-test. The $Z^{\prime}$-factor is a statistic value designed to reflect the dynamic range of the assay as well as the variation associated with the signal measurements. Because the $\mathrm{Z}^{\prime}$-factor is dimensionless, it can be used to compare similar assays (18). The equation and the interpretation of possible Z-values are summarized in Equation 1. To determine the $Z^{\prime}$-factor, the assay was run in a 384-well plate, but only 96 of the 384 wells were used. Each plate had 48 negative control wells and 48 HIV-1-infected wells, arranged in an alternating pattern. Both the HIV-1 NL43-infected positive wells and the negative control wells were treated with $0.1 \mu \mathrm{L}$ dimethyl sulfoxide (DMSO), a typical carrier substance used during drug screens. The plates were analyzed for EGFP expression every $24 \mathrm{~h}$ for a total of $120 \mathrm{~h}$. Maximal levels of fluorescence in the infected wells for both viruses were achieved after 96 h. At this time point, the $Z^{\prime}$-factor for the infectivity assay was determined as 0.78 , characterizing the assay as very robust in this HTS assay format (Figure 4). Infectivity assays can be performed in the presence or absence of agents such as DEAE-dextran that assist infection efficiency by neutralizing cell surface charges. The presence of these agents was found to improve the EGFP signal resulting from the infection by $20 \%$ on average, depending on the virus strain, and did not interfere with the effect of any of the tested established HIV-1 compounds (T-20, TAK779, indinavir, 3TC; data not shown). Screening the plate immediately after plate loading allows for the identification of autofluorescent compounds

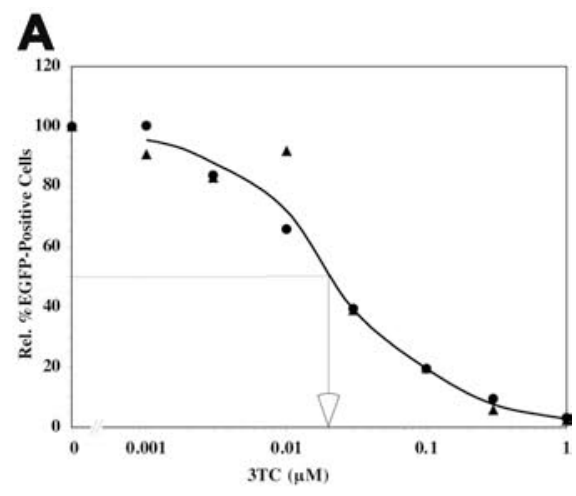

B

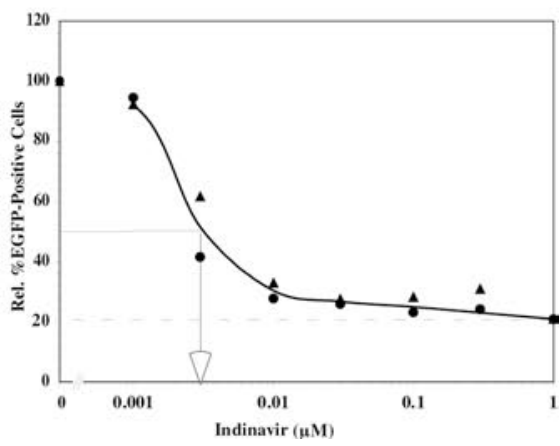

Figure 3. Quantification of reverse transcriptase and protease inhibitor activity using JLTRG-R5 cells. (A) JLTRG-R5 cells were infected at an MOI of 0.01 with HIV-1 NL4-3 in 96-well plates in the presence of increasing concentrations of the HIV-1 reverse transcriptase inhibitor 3TC $(0.001-1 \mu \mathrm{M})$. After 4 days, the samples were analyzed for EGFP expression using flow cytometry. The graphic displays the results of two independent experiments. (Data are normalized to the percentage of infection in untreated cell cultures.) (B) JLTRG-R5 cells were infected at an MOI of 0.1 with HIV-1 NL4-3 in 96-well plates in the presence of increasing concentrations of the HIV-1 protease inhibitor indina$\operatorname{vir}(0.001-1 \mu \mathrm{M})$. After 4 days, the samples were analyzed for EGFP expression using flow cytometry. The graphic displays the results of two independent experiments. The dashed line indicates the level of baseline infection that cannot be inhibited by a protease inhibitor in this experimental setup. Rel. \%EGFP-Positive Cells: relative percentage of EGFP-positive cells normalized to untreated HIV1-infected cultures. MOI, multiplicity of infection; HIV-1, human immunodeficiency virus type 1; EGFP, enhanced green fluorescent protein. 
because no HIV-1-specific EGFP signal is present at this time. A counterscreen for compound cytotoxicity can be performed using cell lines that have been retrovirally transduced with EGFP under the control of a constitutively expressing promoter. A particularly interesting promoter for the generation of such cell lines is the murine stem cell virus (MSCV) promoter. In our hands, transduction of Jurkat cells with an MSCV-EGFP vector results in a 10-fold induction of EGFP expression above background, and the promoter remains unresponsive to stimulation with activating agents such as phorbol 12-myristate13-acetate (PMA; data not shown). However, alternatively established methods such as the addition of alamarBlue ${ }^{\mathrm{TM}}$ (Biosource International) to the wells allow for the direct detection of potential cytotoxicities.

\section{Determination of HIV-1 Entry Inhibitor Effects Using JLTRG-R5 Cells in a 384-Well Plate Format}

To provide proof that JLTRG-R5 cells can be used in the HTS-relevant 384-well format to identify novel HIV entry inhibitors, we quantified the ability of the established HIV-1 entry inhibitor T-20 and the CCR5 inhibitor TAK-779 to block entry of an X4 (NL4-3) and an R5 virus (BAL) in this assay format (Figure 5A). T-20 is a

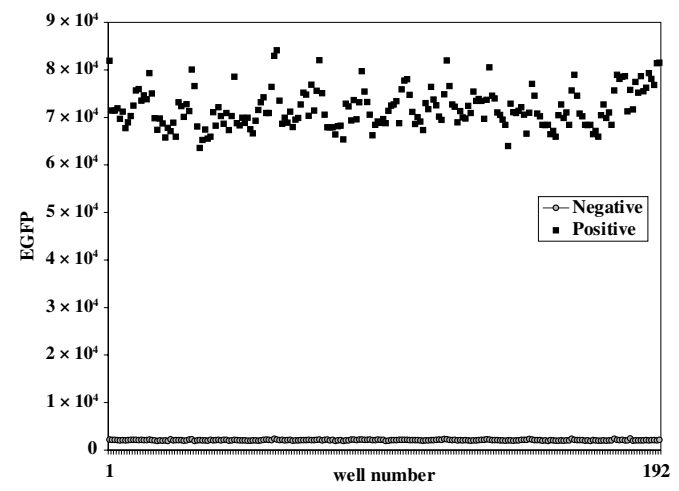

Figure 4. $Z^{\prime}$-test in a 384-well plate-based format. JLTRG-R5 cells were loaded in a 96-well section of a 384-well plate. Two 24-well quadrants were then infected with HIV-1 NL43 (HIV-1 infected, black squares), and two quadrants remained uninfected as background controls (gray squares). HIV-1 expression as indicated by the level of EGFP fluorescence was determined after $96 \mathrm{~h}$ using a Synergy HT Multi-Detection Microplate Reader. HIV-1, human immunodeficiency virus type 1; EGFP, enhanced green fluorescent protein. 36-amino acid peptide corresponding to sequences in the HR-2 domain of gp41 (HXB2 is the reference strain for this sequence). T-20 inhibits HIV-1 entry into cells presumably by binding to HR-1 and preventing the molecular interactions between HR-1 and HR-2 that lead to fusion (19). NL4-3(DIV) contains a naturally occurring variation at position 36 (Asp) from the more commonly observed Gly (reference compendium). Changes at position 36 (e.g., Gly to Asp) such as those seen in NL4-3(GIV) have been associated with increased susceptibility to T-20 in clinical trials (15). T-20 inhibited NL43(DIV), the original T-20-resistant clone, and NL4-3(GIV) and BAL infections at an $\mathrm{IC}_{50}$ of $0.1,0.006$, and 0.005 $\mu \mathrm{g} / \mathrm{mL}$, respectively. These $\mathrm{IC}_{50} \mathrm{~s}$ are consistent with previously published reports (20). TAK-779, a nonpeptide CCR5 antagonist, was efficient at inhibiting HIV-1 BAL infection (R5) but not HIV-1 NL4-3 infection (X4), as expected (21). The $\mathrm{IC}_{50}$ for TAK-779 with respect to HIV-1 BAL inhibition was determined to be $0.005 \mu \mathrm{M}$, again in agreement with previously published data for PBMCs (Table 1) (21). These data suggest that JLTRG-R5 cells allow for the direct identification of protease, reverse transcriptase, integrase, Tat, and entry/fusion inhibitors, as well as neutralizing antibodies, in a 384 -well plate-based format. For the screening of all these inhibitors except for the identification of protease inhibitors, which requires multiple rounds of infection, pseudotyped viruses can be used with comparable efficiency (data not shown).

\section{Determination of the Neutralizing Capacity of Anti-HIV-1 Antibodies}

The idea of providing HIV-1 protection through the application or generation of efficiently neutralizing anti-HIV-1 antibodies has a long tradition. To investigate whether JLTRG-R5 cells could also be used to determine the capacity of anti-HIV-1 antibodies to neutralize HIV-1, we titrated serum from HIV-1- infected patients that had been previously demonstrated to have neutralizing capacity on JLTRG-R5 cells and then infected the cells with either HIV-1 NL4-3 or HIV-1 BAL. Again, the experiments were performed in 384well plates to test whether JLTRG-R5 cells would provide a suitable tool to also screen for such antibodies. For this purpose, we pretreated HIV-1 NL4-3 or BAL supernatants for $1 \mathrm{~h}$ with various concentrations of serum from five HIV-1-infected patients $(0.01-10 \mu \mathrm{L})$ and then transferred the supernatants to the JLTRG-R5 cultures. As positive controls, JLTRG-R5 cells were infected with viral supernatants that had been treated with serum from a healthy donor (Figure 5, B and C). Using the established cell line JC53, the sera KIMA, SMST, SHRO, and SPKE had been previously shown to be highly neutralizing, whereas YOAL showed only low reactivity (13). These results could be reproduced exactly using JLTRG-R5 cells, demonstrating the feasibility of JLTRG-R5 cells to be used in assays that characterize the ability of patient sera to neutralize HIV-1 infection.

\section{DISCUSSION}

Due to the inherent mutagenic nature of HIV-1 resulting in the rapid acquisition of HIV-1 mutations conferring resistance, there is overwhelming pressure for the development of new pharmaceutical HIV-1 inhibitors in order to maintain the current success of HAART. To this end, here we introduce a T-cell-based reporter system designed for the identification of novel HIV-1 inhibitors, suitable for HTS in a platebased 384-well format. In addition, we demonstrate that the assay can be utilized to quantify the neutralizing capacity of anti-HIV-1 antibodies, thus providing a powerful tool to assist HIV1 vaccine research.

Because fluorescent proteins can be directly detected and quantified in cell culture without any further manipulation during the assay quantification step, they provide an ideal tool to establish HTS assays. However, generally the achievable levels of EGFP expression in reporter assays described to date are too low to produce a sufficiently high 


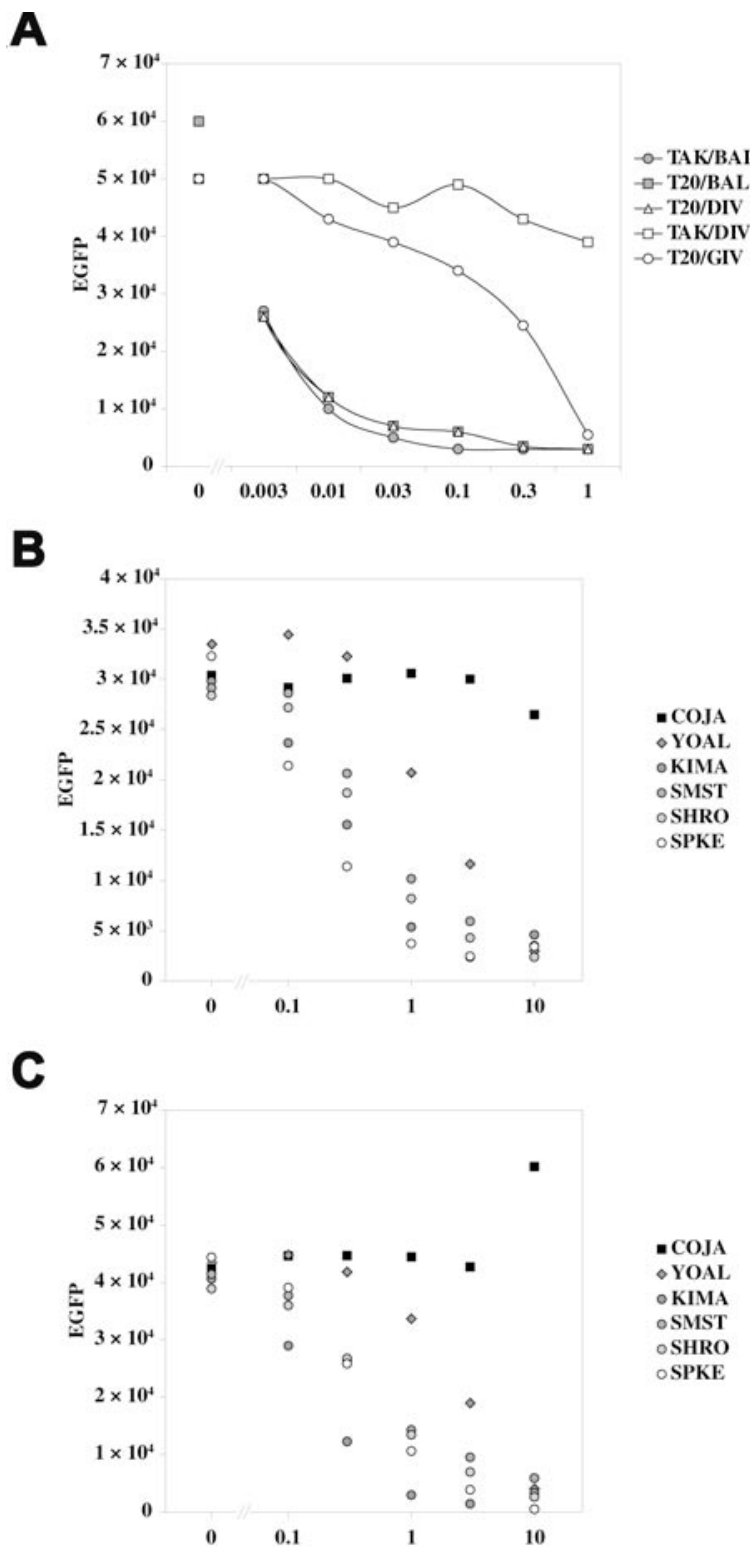

Figure 5. Inhibition of HIV-1 entry. In a 384-well plate, JLTRGR5 cells were infected with either HIV-1 NL4-3(GIV) (T-20 resistant; X4) NL4-3(DIV) (T-20 sensitive; X4), or HIV-1 BAL (R5) in the presence of various concentrations of the fusion inhibitor T-20 (0.003-1 $\mu \mathrm{g} / \mathrm{mL}$ ) or the CCR5 antagonist TAK-779 (TAK; 0.003-1 $\mu \mathrm{M}$ ). EGFP fluorescence as a quantitative marker for HIV-1 expression was measured after 3 days using plate-based fluorometry. Infectious viral culture supernatants containing (A) HIV-1 BAL or (B) HIV-1 NL43 were pretreated for $1 \mathrm{~h}$ with various amounts of serum from five HIV-1 patients and one noninfected volunteer $(0-10 \mu \mathrm{L})$. Patient sera KIMA, SMST, SHRO, and SPKE had been previously determined as highly neutralizing, whereas the patient serum YOAL has low reactivity. The serum COJA originates from a healthy volunteer. The serum-treated infectious supernatants were then added to the JLTRG$\mathrm{R} 5$ cells that had been seeded at a density of $1 \times 10^{5}$ cells per 384-well plate (total volume $100 \mu \mathrm{L}$ ). The plates were analyzed for EGFP fluorescence 4 days postinfection. HIV-1, human immunodeficiency virus type 1; EGFP, enhanced green fluorescent protein. signal-to-noise ratio for a plate-based analysis format, thus rendering these current assays not truly amenable to efficient HTS. For this reason, assays based on SEAP or luciferase activity, which generally have a high dynamic range, are commonly used. In fact, the two most advanced reporter cell lines, MOCHA (10) and CEM.NKR-CCR5 (11), which are presently available to identify and study novel HIV-1 inhibitors, take advantage of these quantitative markers. Nevertheless, the use of these cell lines has considerable disadvantages because both indicator genes require additional manipulation and reagents during assay analysis, which render the readout more error prone and timeconsuming and substantially increase the costs during an HTS. In addition, experimental setups that require the transfer of HIV-1containing supernatants increase the biohazard generally associated with working with HIV-1.

With the establishment of the JLTRG-R5 reporter cell line, we demonstrate that it is possible to overcome these limitations and develop a $\mathrm{CCR}^{+}{ }^{+} \mathrm{CXCR} 4^{+}$reporter T-cell line that delivers an unusually high EGFP signal upon HIV-1 Tat expression or HIV-1 infection and can be used for drug screening in a 384-well plate-based format with a $Z^{\prime}$-factor of 0.78 . Due to the stable nature of the cells, which carry an integrated LTR-
EGFP reporter plasmid, any assay using JLTRG-R5 cells requires no manipulation during assay preparation (e.g., transfection of reporter plasmids) or assay analysis. The usage of EGFP as a direct and quantitative marker of HIV-1 expression permits the continuous realtime observation of the influence of putative HIV-1-inhibiting compounds on HIV-1 replication over an extended period of time, allowing us to study not only the cumulative outcome but also to observe potential differences in the inhibitory onset kinetics of various test compounds (22). In addition, in contrast to infectivity assays that do not use EGFP as a readout, JLTRG-R5 cells can be utilized for the direct investigation of the underlying mechanism at the single-cell level using flow cytometric analysis. This feature previously had been established in CEMGFP cells, but because these cells do not express CCR5, they are not susceptible to infection with R5 viruses and provide a poor screening tool for HIV-1 entry inhibitors (23). In addition, in our hands, CEM-GFP cells have a high, constitutive EGFP background (one log-shift in flow cytometric analysis) when compared with the parental CEM cells, thereby significantly decreasing the dynamic range following HIV-1 infection. In fact, the strict correlation of EGFP fluorescence and HIV-1 expression/replication in JLTRG-R5 cells allows for the acquisition of additional data at the end of the drug screen by selectively collecting samples from interesting wells and subjecting them to flow cytometric analysis. By this means, data on, for example, cell viability, apoptosis, or activation markers can be immediately obtained. Because JLTRG-R5 cells are susceptible to infection with a wide variety of patient isolates, the cells can then be used for detailed verification analysis.

We obtained optimal readout after $96 \mathrm{~h}$. This incubation period may, similar to other infectivity assays, impose a limitation with respect to the successful identification of highly instable but potentially inhibitory compounds. However, due to the relatively short half-life of the infectious viral particles in the inoculum, this should only affect the detection of extremely short-lived HIV-1 entry/ 
fusion inhibitors. For other HIV-1 inhibitors (e.g., reverse transcriptase or protease inhibitors), compound stability has a greater influence on the sensitivity of the assay; however, the delay in viral replication that is caused by the initial presence of the unstable compound will still allow for the detection of a compound with a reasonably short half-life and would justify advancement to a drug development state. A second limitation that is generally shared among cell-based assays is imposed by the potential cytotoxicity of the carrier substance (e.g., DMSO), in which the compounds are dissolved. JLTRG-R5 cells tolerate a DMSO concentration of up to $0.5 \%$ DMSO. Higher DMSO concentrations activate the cells, which may adversely influence a drug screening effort. The tolerance of the cells with respect to the carrier substance defines the upper limit for the utilized compound concentration. In a situation where higher drug concentrations are desirable, molecular target screens are clearly preferable.

Notably, the $\mathrm{IC}_{50}$ data for the HIV1 reverse transcriptase inhibitor 3TC, the protease inhibitor indinavir, and the entry/fusion inhibitors TAK-779 and T-20 obtained in either experimental format compare very well with data previously obtained for these drugs $(19,21)$. This ability of JLTRG-R5 cells to reproduce data previously obtained on PBMCs and the convenience of EGFP as a direct and quantitative readout for HIV-1 expression make JLTRG-R5 cells a very powerful and unique tool that should greatly enhance our ability to identify novel HIV-1 inhibitors.

\section{ACKNOWLEDGMENTS}

This work was supported, in part, through the Developmental Core of the University of Alabama (UAB) Center for AIDS Research (CFAR) (to C.O.J.), National Institutes of Health (NIH) grant no. P30 AI27767, and through the UAB Mucosal HIV and Immunology Center (MHIC), NIH grant no. DK-64400, and NIH grant no. NS050810-01 (to O.K). We gratefully acknowledge F. Mammano for the generous gift of proviral plasmids T28-
X4-2 and T28-R5-2. We would like to thank J.C. Kappes for valuable discussion and encouragement and $M$. Spell for technical assistance with flow cytometric analysis.

\section{COMPETING INTERESTS STATEMENT}

The authors declare no competing interests.

\section{REFERENCES}

1. Blankson, J.N., D. Persaud, and R.F. Siliciano. 2002. The challenge of viral reservoirs in HIV-1 infection. Annu. Rev. Med. 53:557-593.

2. Menendez-Arias, L. 2002. Targeting HIV: antiretroviral therapy and development of drug resistance. Trends Pharmacol. Sci. 23:381-388

3.O'Brien, W.A., M. Sumner-Smith, S.H. Mao, S. Sadeghi, J.Q. Zhao, and I.S. Chen. 1996. Anti-human immunodeficiency virus type 1 activity of an oligocationic compound mediated via gp120 V3 interactions. J. Virol. 70:2825-2831.

4.Doranz， B.J., K. Grovit-Ferbas, M.P. Sharron, S.H. Mao, M.B. Goetz, E.S. Daar, R.W. Doms, and W.A. O'Brien. 1997. A small-molecule inhibitor directed against the chemokine receptor CXCR4 prevents its use as an HIV-1 coreceptor. J. Exp. Med. 186:1395-1400.

5. Kira, T., J.P. Merin, M. Baba, S. Shigeta, and T. Okamoto. 1995. Anti-Tat MTT assay: a novel anti-HIV drug screening system using the viral regulatory network of replication. AIDS Res. Hum. Retroviruses 11:1359-1366.

6. Witvrouw, M., R. Pauwels, A.M. Vandamme, D. Schols, D. Reymen, N. Yamamoto, J. Desmyter, and E. De Clercq. 1992. Cell type-specific anti-human immunodeficiency virus type 1 activity of the transactivation inhibitor Ro5-3335. Antimicrob. Agents Chemother. 36:2628-2633.

7.Daelemans, D., E. De Clercq, and A.M. Vandamme. 2001. A quantitative GFPbased bioassay for the detection of HIV-1 Tat transactivation inhibitors. J. Virol. Methods 96:183-188.

8. Del Rosario, M., J.C. Stephans, J. Zakel, J. Escobedo, and K. Giese. 1996. Positive selection system to screen for inhibitors of human immunodeficiency virus-1 transcription. Nat. Biotechnol. 14:1592-1596.

9. Rice, A.P. and M.B. Mathews. 1988. Transcriptional but not translational regulation of HIV-1 by the tat gene product. Nature 332:551-553.

10.Miyake, H., Y. Iizawa, and M. Baba. 2003. Novel reporter T-cell line highly susceptible to both CCR5- and CXCR4-using human immunodeficiency virus type 1 and its application to drug susceptibility tests. J. Clin. Microbiol. 41:2515-2521.
11.Spenlehauer, C., C.A. Gordon, A. Trkola and J.P. Moore. 2001. A luciferase-reporter gene-expressing T-cell line facilitates neutralization and drug-sensitivity assays that use either R5 or X4 strains of human immunodeficiency virus type 1. Virology 280:292-300.

12.Kutsch, O., D.N. Levy, P.J. Bates, J. Decker, B.R. Kosloff, G.M. Shaw, W. Priebe, and E.N. Benveniste. 2004. Bis-anthracycline antibiotics inhibit human immunodeficiency virus type 1 transcription. Antimicrob. Agents Chemother. 48:1652-1663.

13.Wei, X., J.M. Decker, S. Wang, H. Hui, J.C. Kappes, X. Wu, J.F. Salazar-Gonzalez M.G. Salazar, et al. 2003. Antibody neutralization and escape by HIV-1. Nature 422:307312.

14.Schmid, I., W.J. Krall, C.H. Uittenbogaart, J. Braun, and J.V. Giorgi. 1992. Dead cell discrimination with 7-amino-actinomycin $\mathrm{D}$ in combination with dual color immunofluorescence in single laser flow cytometry. Cytometry 13:204-208.

15.Wei, X., J.M. Decker, H. Liu, Z. Zhang, R.B. Arani, J.M. Kilby, M.S. Saag, X. Wu, et al. 2002. Emergence of resistant human immunodeficiency virus type 1 in patients receiving fusion inhibitor (T-20) monotherapy. Antimicrob. Agents Chemother. 46:18961905.

16.Schinazi, R.F., R.M. Lloyd, Jr., M.H. Nguyen, D.L. Cannon, A. McMillan, N. Ilksoy, C.K. Chu, D.C. Liotta, et al. 1993 Characterization of human immunodeficiency viruses resistant to oxathiolane-cytosine nucleosides. Antimicrob. Agents Chemother. 37:875-881.

17.Vacca, J.P., B.D. Dorsey, W.A. Schleif, R.B. Levin, S.L. McDaniel, P.L. Darke, J. Zugay, J.C. Quintero, et al. 1994. L-735,524: an orally bioavailable human immunodeficiency virus type 1 protease inhibitor. Proc. Natl. Acad. Sci. USA 91:4096-4100.

18.Zhang, J.H., T.D. Chung, and K.R. Oldenburg. 1999. A simple statistical parameter for use in evaluation and validation of high throughput screening assays. J. Biomol. Screen. 4:67-73.

19.Wild, C., T. Greenwell, and T. Matthews. 1993. A synthetic peptide from HIV-1 gp41 is a potent inhibitor of virus-mediated cell-cell fusion. AIDS Res. Hum. Retroviruses 9:10511053

20.Wild, C.T., D.C. Shugars, T.K. Greenwell, C.B. McDanal, and T.J. Matthews. 1994 Peptides corresponding to a predictive alphahelical domain of human immunodeficiency virus type $1 \mathrm{gp} 41$ are potent inhibitors of virus infection. Proc. Natl. Acad. Sci. USA 91:9770-9774.

21.Baba, M., O. Nishimura, N. Kanzaki, M. Okamoto, H. Sawada, Y. Iizawa, M. Shiraishi, Y. Aramaki, et al. 1999. A smallmolecule, nonpeptide CCR5 antagonist with highly potent and selective anti-HIV-1 activity. Proc. Natl. Acad. Sci. USA 96:5698-5703.

22.Ferguson, N.M., C. Fraser, and R.M. Anderson. 2001. Viral dynamics and antiviral pharmacodynamics: rethinking in vitro measures of drug potency. Trends Pharmacol. Sci. 22:97-100. 
23.Gervaix, A., D. West, L.M. Leoni, D.D. Richman, F. Wong-Staal, and J. Corbeil.

1997. A new reporter cell line to monitor HIV infection and drug susceptibility in vitro. Proc.

Natl. Acad. Sci. USA 94:4653-4658.

Received 6 June 2005; accepted 16 September 2005.

Address correspondence to:

Olaf Kutsch

Division of Hematology/Oncology

University of Alabama at Birmingham

KAUL 840

720 20th Street South

Birmingham, AL 35294, USA

e-mail:okutsch@uab.edu

To purchase reprints

of this article, contact

Reprints@BioTechniques.com 